\title{
Significance of Anti-HLA Antibodies on Adult and Pediatric Heart Allograft Outcomes
}

\author{
Massimo Mangiola ${ }^{1 *}$, Marilyn Marrari ${ }^{1}$, Brian Feingold ${ }^{2}$ and Adriana Zeevi ${ }^{1 *}$ \\ ${ }^{1}$ Division of Transplant Pathology, Department of Pathology, University of Pittsburgh Medical Center, Pittsburgh, PA, USA, \\ ${ }^{2}$ Pediatric Cardiology, The Children's Hospital of Pittsburgh of UPMC, Pittsburgh, PA, USA
}

OPEN ACCESS

Edited by:

Narinder K. Mehra

All India Institute of

Medical Sciences, India

Reviewed by:

Stanislaw Stepkowski,

University of Toledo, USA

Reem Al-Daccak,

French Institute of Health and

Medical Research, France

*Correspondence:

Massimo Mangiola

mangiolam@upmc.edu;

Adriana Zeevi

zeevia@upmc.edu

Specialty section:

This article was submitted to Alloimmunity and Transplantation,

a section of the journal

Frontiers in Immunology

Received: 13 September 2016

Accepted: 03 January 2017

Published: 27 January 2017

Citation:

Mangiola M, Marrari M, Feingold B and Zeevi A (2017) Significance of Anti-HLA Antibodies on Adult and Pediatric Heart Allograft Outcomes.

Front. Immunol. 8:4.

doi: 10.3389/fimmu.2017.00004
As methods for human leukocyte antigens (HLA) antibody detection have evolved and newer solid phase assays are much more sensitive, the last 15 years has seen a renewed focus on the importance of HLA antibodies in solid organ transplant rejection. However, there is still much controversy regarding the clinical significance of antibody level as depicted by the mean fluorescence intensity of a patient's neat serum. Emerging techniques, including those that identify antibody level and function, show promise for the detection of individuals at risk of allograft rejection, determination of the effectiveness of desensitization prior to transplant, and for monitoring treatment of rejection. Here, we review current publications regarding the relevance of donor-specific HLA antibodies (DSA) in adult and pediatric heart transplantation $(\mathrm{HT})$ with graft survival, development of antibody-mediated rejection and cardiac allograft vasculopathy (CAV). The negative impact of DSA on patient and allograft survival is evident in adult and pediatric HT recipients. Many questions remain regarding the most appropriate frequency of assessment of pre- and posttransplant DSA as well as the phenotype of DSA memory vs. true de novo antibody using large multicenter adult and pediatric cohorts and stateof-the-art methodologies for DSA detection and characterization.

Keywords: AMR, heart transplantation, donor-specific antibodies, allograft vasculopathy, graft failure

\section{INTRODUCTION}

Heart transplantation (HT) has become an accepted therapy for adult and pediatric patients with end-stage heart failure. Despite improved immunosuppression regimens, rejection remains the most common cause of death in the first 5 years after HT. Both cellular and humoral immunemediated processes that can damage the allograft are primarily directed against human leukocyte antigens (HLA). Antibodies against HLA can be found in patients prior to transplantation after exposure to foreign HLA through blood transfusion, pregnancy, previous transplant, and use of homograft tissue during surgery for some congenital heart defects. Ventricular assist devices (VAD) have also been implicated in the development of HLA antibodies, termed allosensitization. Exposure to donor HLA after HT may also induce de novo production of donor-specific HLA antibodies (DSA). The impact of circulating HLA antibodies on heart allografts has been the focus of many investigations and reviews. The introduction of solid phase assays (SPA) based on the luminex single antigen bead assay (SAB) has improved the 
sensitivity and specificity of HLA antibody detection; however, it also introduced new challenges for assay interpretation and determining its clinical relevance (1).

Identification of DSA enables the clinician to make informed decisions regarding acceptance of the organ and the choice of immunosuppression (2). Presence of DSA is not always considered a contraindication but rather a risk factor for organ transplantation success (3). Optimizing transplantation of allosensitized candidates is challenging and program specific. The main challenge with the new SPA technology is decision-making regarding donor organ acceptance based solely on antibody strength determined by mean fluorescence intensity (MFI) (2-5). The threshold for accepting a donor for a sensitized patient may vary depending on the patient's clinical status, antibody level, and protocols available for antibody removal therapy. Considering the SPA modification to detect complement-fixing antibodies (C1q-SAB) has reduced the estimated incompatible donor pool in highly sensitized patients (6). Optimizing transplantation of allosensitized candidates using SAB and C1q-SAB methodology to prioritize the assignment of unacceptable antigens has allowed transplantation of highly allosensitized patients across the DSA barrier with survival rates comparable to DSA- heart transplant recipients (5).

Titration of sera prior to $\mathrm{SAB}$ testing has emerged as a more accurate way to assess the true level of DSA as compared to MFI value of undiluted sera (7). Furthermore, titration studies provide better estimates of responsiveness to antibody removal therapies (8).

Recognition that some preformed antibodies are against denatured HLA antigens with very little clinical relevance may also impact the search for an acceptable donor $(4,9)$. The assignment of unacceptable antigens has been greatly improved also by incorporating patterns of epitope reactivity and history of sensitizing events. Recognizing the limitations and advantages of current available methods for antibody determination, quantitation and function has facilitated the introduction of the virtual crossmatch (VXM) in thoracic transplantation. Previously, the need for prospective crossmatch (XM) in sensitized patients was associated with longer waitlist duration and increased mortality (10). Although VXM is widely used for organ allocation, its validity highly depends on how accurate and current is the information on patient sensitization events and comprehensive DNA-based HLA typing of prospective donors as antibodies can be made against every possible polymorphic HLA target antigen (2-5).

\section{RELEVANCE OF DSA ON OUTCOMES}

In this report, we focus on a short review of the current stateof-the-art regarding the role of DSA in adult and pediatric HT as determined by the following outcome measures: graft survival (GS), development of antibody-mediated rejection (AMR), and cardiac allograft vasculopathy (CAV) (Tables 1 and 2). Although we limit this review to the last 6 years, the retrospective nature of some studies may influence the relevance of DSA on clinical outcomes due to the use of less sensitive testing methods. Furthermore, we considered separately the role of DSA on adult and pediatric clinical outcomes to highlight potential similarities and differences in the two cohorts.

\section{ADULT HT}

\section{Graft Survival}

The prevalence of allosensitization in heart transplant candidates increased with the introduction of SPA for screening for HLA antibodies (11) (Table 1). Nevertheless, the risk for poor GS has remained a significant finding even in the more sensitive SPA testing era $(11,12)$. The presence of non-cytotoxic HLA antibodies identified by SAB was associated with high risk of death, early graft failure, and late cellular- and antibody-mediated rejection; these findings underscore the need for using sensitive Luminex platform SPA to accurately determine the presence of circulating HLA antibodies (12). Detection of Class I DSA pretransplant was a predictor of short-term but not long-term survival as compared to non-DSA (13). In this study limiting the testing on pretransplantation, the authors could not identify the impact of persistent vs. transient DSA and of de novo DSA on clinical outcomes.

De novo antibody production and its role in cardiac allograft survival has been described in several studies (14-16). In a retrospective adult cohort, de novo DSA was associated with poor patient survival ( $\mathrm{HR}=3.198)$, while de novo and persistent DSA was worst $(\mathrm{HR}=4.351)(14)$. Similarly, patients with persistent de novo, mostly Class II DQ-specific DSA, had worse survival (15). The 15-year survival was highest in patients who never developed DSA vs. those that developed DSA posttransplantation (70 vs. $47 \%$ ), and patients with late de novo DSA appearing more than 1 year post transplantation had poorest survival (16). Thus, determining the presence of DSA pretransplant for risk assessment and monitoring for persistent and de novo DSA posttransplant provide the most comprehensive information for clinical management.

\section{Antibody-Mediated Rejection}

The challenges of AMR diagnosis post HT have been addressed by many single-center studies and consensus conferences. In the current era, diagnosis of this clinically important entity has been improved by standardized classification of histologic and immunologic changes in endomyocardial biopsies (EMB) (17) and by advances in the detection of HLA antibodies. Although not required for diagnosis of pathologic AMR (pAMR), the detection of HLA antibodies pre- and posttransplantation has been helpful for risk stratification for the development of AMR and for guiding treatment strategies (18). Patients with positive VXM defined in the presence of DSA >1,500 MFI by SAB had a higher incidence of AMR and cell-mediated rejection. Similar outcomes were observed with positive flow crossmatch (FXM) suggesting that SAB MFI $>1,500$ can be used as surrogate for FXM (19). Increased risk for a positive complement-dependent cytotoxicity (CDC) XM and early AMR was observed in patients with persistent $\mathrm{C} 1 \mathrm{q}+$ DSA (20). However, patients who had DSA but lost the C1q reactivity posttransplant did not develop early $A M R$, and the strength of neat sera on $\mathrm{SAB}$ did not predict $\mathrm{C} 1 \mathrm{q}$ reactivity. In contrast, high titer DSA $(>1: 16)$ has been associated with 
TABLE 1 | Cited publications from the last 6 years (2010-present) showing the impact of HLA antibody on heart transplantation in adult recipients

\begin{tabular}{|c|c|c|c|c|c|c|c|}
\hline Reference & $\begin{array}{l}\text { Number of patients } \\
\text { (study period) }\end{array}$ & Method & DSA & GS & AMR & CAV & Comments \\
\hline $\begin{array}{l}\text { Gandhi } \\
\text { et al. (19) }\end{array}$ & $\begin{array}{l}85 \text { (August 2006- } \\
\text { January 2010) }\end{array}$ & $\begin{array}{l}\text { CDC-AHG PRA/XM, } \\
\text { Flow XM, SAB }\end{array}$ & $\begin{array}{l}\text { All CDC XM-; DSA+ (MFI } \\
>1,500), n=11(13 \%): \\
\text { Class I =2, Class } \|=6 \\
\text { Class I }+\|=3\end{array}$ & & $\begin{array}{l}(n=80 \text { for biopsy) } \\
\text { AMR: } 7 / 11 \text { DSA+ }\end{array}$ & & $\begin{array}{l}\mathrm{CMR} \geq 1 \mathrm{R} / 1 \mathrm{~A}: \text { 9/11 DSA+vs. } \\
48 / 69 \mathrm{DSA}-/ \text { weak; DSA MFI } \\
>1,500 \text { associated with increased } \\
\text { incidence of AMR and CMR }\end{array}$ \\
\hline Smith et al. (14) & $\begin{array}{l}243 \text { (October 1995- } \\
\text { July 2004) }\end{array}$ & $\mathrm{SAB}(8.8 \pm 2.5)$ & $\begin{array}{l}57 \text { dnDSA } \\
\text { Class } \|=48(42 / 48 \mathrm{DQ})\end{array}$ & $\begin{array}{l}\text { Poor GS } \\
p=0.0001(H R=4.35)\end{array}$ & & $\begin{array}{l}29 \% 5 y ; 55 \% \\
10 y\end{array}$ & DnDSA risk for poor GS and CAV \\
\hline Ho et al. (16) & $\begin{array}{l}950 \text { (January 1995- } \\
\text { December 2009) }\end{array}$ & $\begin{array}{l}\text { CDC T and B, SAB } \\
\text { (mean number of } \\
\text { sera tested per } \\
\text { patient }=24 \pm 9 \text { ) }\end{array}$ & $\begin{array}{l}221 \text { dnDSA } 1 \text { y, } 118 \text { dnDSA } \\
>1 \text { y, } 460 \text { no HLA-Ab }\end{array}$ & $\begin{array}{l}\text { GS } 52 \%, p<0.005 \\
\text { GS } 48 \%, p<0.001 \\
\text { GS } 70 \%\end{array}$ & 23 & & $\begin{array}{l}\text { DSA and non-DSA increased in } \\
\text { rejection }\end{array}$ \\
\hline Loupy et al. (29) & 196 (1985-2009) & $\mathrm{SAB}$ & $\begin{array}{l}20 \text { very late rejection (VLR } \\
>7 \mathrm{y})\end{array}$ & & & $\begin{array}{l}\text { CAV grade VLR, } \\
2.06 \text { vs. } 0.76 \text { in } \\
\text { control }\end{array}$ & VLR associated with severe CAV \\
\hline $\begin{array}{l}\text { Hodges et al. } \\
\text { (15) }\end{array}$ & $\begin{array}{l}762 \text { (November 2005- } \\
\text { August 2011) }\end{array}$ & $\begin{array}{l}\text { Luminex } \\
\text { Screen, SAB }\end{array}$ & $15 \operatorname{AMR}(14 / 15$ dnDSA) & $\begin{array}{l}1.8 \text { y mean survival } \\
\text { after AMR treatment }\end{array}$ & 15 & & Late cardiac AMR with dnDSA \\
\hline Zeevi et al. (20) & $\begin{array}{l}15 \text { (8 pediatric, } 7 \\
\text { adult) }\end{array}$ & SAB, SAB-C1q & $\begin{array}{l}35 \text { DSA in } 14 \text { patients: } \\
\text { Class } I=4, \text { Class } \|=2 \text {, } \\
\text { Class } I+\|=8\end{array}$ & & $\begin{array}{l}\text { 1st month post-Tx: } \\
7 / 7 \text { cAMR+ are } \\
\text { DSA+/C1q+; } 4 \\
\text { cAMR-free, DSA+/ } \\
\text { C1q- }(p<0.005)\end{array}$ & & $\begin{array}{l}\text { Persistent C1q+ DSA post-Tx } \\
\text { associated with early clinical AMR }\end{array}$ \\
\hline $\begin{array}{l}\text { Potena } \\
\text { et al. (11) }\end{array}$ & $173(2000-2005)$ & $\begin{array}{l}\text { CDC/PRA, Luminex } \\
\text { Screen }\end{array}$ & $\begin{array}{l}\text { Pre-Tx } 32 \mathrm{Ab}+\text { Class } I=28, \\
\text { Class } \|=16, \text { Class } I+\|=12\end{array}$ & $\begin{array}{l}\text { Survival } \\
65 \% \text { for } \mathrm{Ab}+ \\
82 \% \text { for } \mathrm{Ab}-\end{array}$ & $\begin{array}{l}\text { 9/37 with biopsy were } \\
\text { HLA-Ab+, pAMR }>2\end{array}$ & & \\
\hline Raess et al. (13) & $272(1989-2010)$ & $\begin{array}{l}\text { CDC-PRAXXM, } \\
\text { Luminex screen, SAB, } \\
\text { SAB-C19 }\end{array}$ & $\begin{array}{l}\text { DSA } 26(9.6 \%), \text { Class } I=14 \\
\text { Class } \|=5, \text { Class } I+\|=7 \\
\text { C1q+ DSA }=2\end{array}$ & $\begin{array}{l}\text { Overall survival: } 80 \% \\
\text { (1 y), } 68 \% \text { (5 y) } \\
\text { SAB Class I DSA+: } \\
62 \% \text { (1 y), } 50 \%(5 \text { y) } \\
\text { SAB Class I DSA-: } \\
87 \% \text { (1 y), } 73 \%(5 \text { y) }\end{array}$ & $\begin{array}{l}\text { Fatal pAMR }=6 \text {, all } \\
\leq 1 \text { month post- } T x\end{array}$ & $\begin{array}{l}(n=245) \text { CAV- } \\
\text { free survival: } 96 \% \\
(1 \mathrm{y}), 86 \%(5 \mathrm{y})\end{array}$ & $\begin{array}{l}\text { ACR-free survival: } 38 \% \text { ( } 1 \text { y), } \\
30 \% \text { (5 y); pre-Tx HLA Ab status } \\
\text { affected short-term survival but } \\
\text { had no effect on long-term survival/ } \\
\text { rejection }\end{array}$ \\
\hline $\begin{array}{l}\text { Topilsky } \\
\text { et al. (27) }\end{array}$ & $\begin{array}{l}51 \text { (January 2004- } \\
\text { December 2009) }\end{array}$ & $\begin{array}{l}\text { SAB; Flow XM for } 30 \\
\text { patients }\end{array}$ & $\begin{array}{l}\text { All CDC-XM-; DSA + } 17 \\
(33 \%): \text { Class I }=4 \\
\text { Class } \|=11, \text { Class } I+\|=2\end{array}$ & & & $\begin{array}{l}36(71 \%) \text { with } \\
\text { Grade } 1 \text { CAV }\end{array}$ & $\begin{array}{l}\text { CAV analysis done for patients with } \\
\text { only Class II DSA; pre-Tx Class } \\
\text { II DSA may give higher risk of } \\
\text { accelerated CAV: DSA+ } 100 \% \text { vs. } \\
\text { DSA- } 64.2 \% \text { at } 4 \text { y }\end{array}$ \\
\hline Tible et al. (22) & $\begin{array}{l}111 \text { (October 2009- } \\
\text { September 2010) }\end{array}$ & $\begin{array}{l}\text { SAB, } 150 \text { paired DSA } \\
\text { and EMB }\end{array}$ & $\begin{array}{l}47 / 150 \text { DSA }+, \text { Class I }= \\
40.4 \%, \text { Class } \|=40.4 \%, \\
\text { Class I + } \mid=19.2 \%\end{array}$ & & 37 & & $\begin{array}{l}\mathrm{Ml} \text { and CD68 associated with } \\
\text { DSA+ }\end{array}$ \\
\hline Frank et al. (28) & $\begin{array}{l}109 \text { (February 1996- } \\
\text { June 2011) }\end{array}$ & $\begin{array}{l}\text { SAB, } 330 \text { paired DSA } \\
\text { and EMB }\end{array}$ & $\begin{array}{l}\text { 51/112, Class } \mid=5 \\
\text { Class } \|=26, \text { Class } \mid+\|=20\end{array}$ & & & $\begin{array}{l}24(22 \%): 40 \% \\
\text { DSA+, } 13 \% \\
\text { DSA- }\end{array}$ & $\begin{array}{l}33 \% \text { with CAV pre-Tx DSA+; Class } \\
\text { II DSA, IF C4d+, and MI high risk } \\
\text { for failed allograft with CAV }\end{array}$ \\
\hline
\end{tabular}


TABLE 1 | Continued

\begin{tabular}{|c|c|c|c|c|c|c|c|}
\hline Reference & $\begin{array}{l}\text { Number of patients } \\
\text { (study period) }\end{array}$ & Method & DSA & GS & AMR & CAV & Comments \\
\hline $\begin{array}{l}\text { Coutance } \\
\text { et al. (24) }\end{array}$ & $\begin{array}{l}20 \text { (November 2006- } \\
\text { February 2013) }\end{array}$ & Luminex Screen, SAB & 19/20 tested were dnDSA+ & $50 \%$ after 1 y & $\begin{array}{l}\text { Late AMR (>1 y } \\
\text { post-Tx) }\end{array}$ & & $\begin{array}{l}\text { Prognosis for late AMR poor } \\
\text { despite aggressive therapy }\end{array}$ \\
\hline $\begin{array}{l}\text { O'Connor } \\
\text { et al. (12) }\end{array}$ & $\begin{array}{l}\text { 12,858 (June 2004- } \\
\text { March 2013); UNOS } \\
\text { database }\end{array}$ & CDC-PRA, Flow-PRA & $\begin{array}{l}\mathrm{PRA} \geq 10 \%, \text { Class I: } \\
\mathrm{CDC}+=227, \text { Flow }+=2,243, \\
\text { Class II: CDC+ }=126 \\
\text { Flow }+=2,218\end{array}$ & $\begin{array}{l}\mathrm{PRA} \geq 10 \%: \\
\mathrm{HR}=1.24(95 \% \mathrm{Cl} \\
1.12-1.36)\end{array}$ & & & $\begin{array}{l}\text { Percent Ab+ patients increased } \\
\text { from } 2005 \text { to } 2011 \text { as use of flow } \\
\text { increased; pre-Tx PRA } \geq 10 \% \text { by } \\
\text { Flow associated with increased risk } \\
\text { of graft loss }\end{array}$ \\
\hline $\begin{array}{l}\text { Svobodova } \\
\text { et al. (21) }\end{array}$ & $\begin{array}{l}264 \text { (April 2005- } \\
\text { December 2012; } \\
\text { mean follow-up } \\
39 \text { months, range } \\
\text { 19-66) }\end{array}$ & $\begin{array}{l}\text { CDC-PRAXM; SAB, } \\
\text { SAB-C1q }\end{array}$ & $\begin{array}{l}\mathrm{DSA}=28(11 \%): \\
\text { Class } \mathrm{I}=18, \text { Class } \|=3 \\
\text { Class } \mid+\|=7, \mathrm{C} 1 \mathrm{q}+ \\
\mathrm{DSA}=4\end{array}$ & $90 \%$ (1 y), 79\% (5 y) & $19(7 \%)$ & $31(12 \%)$ & $\begin{array}{l}74 \text { patients }(28 \%) \text { with } 83 \text { instances } \\
\text { of ACR grade } \geq \text { Banff } 2 \text {; pre-Tx } \\
\text { DSA and elevated peak CDC-PRA } \\
\text { were strongest predictors of AMR }\end{array}$ \\
\hline Frank et al. (23) & $44(2005-2011)$ & $\begin{array}{l}\text { SAB-C1q paired with } \\
\text { EMB C4d stain }\end{array}$ & $\begin{array}{l}\text { C1q+ DSA in } 82 \% \text { with graft } \\
\text { dysfunction }\end{array}$ & $\begin{array}{l}18 / 44 \text { died or } \\
\text { retransplanted }\end{array}$ & $\begin{array}{l}\text { 16/17 C4d+ IF had } \\
\text { C1q+ DSA; } 24 \text { C1q+ } \\
\text { DSA were C4d-IF }\end{array}$ & & $\begin{array}{l}\text { Better concordance of C4d+ IF } \\
\text { with C1q DSA as compared to } \\
\text { IgG DSA }\end{array}$ \\
\hline Loupy et al. (25) & 40 , failing grafts & $\mathrm{SAB}$ & & & $\mathrm{AMR}=19$ & & \\
\hline $\begin{array}{l}\text { Clerkin } \\
\text { et al. (26) }\end{array}$ & $\begin{array}{l}689 \text { (January 2004- } \\
\text { December 2013, } \\
\text { follow-up through } \\
\text { October 2015) }\end{array}$ & $\begin{array}{l}\text { Luminex SAB and/or } \\
\text { CDC screen }\end{array}$ & $\begin{array}{l}\text { Overall: } n=29(42.6 \%) ; \\
\text { early AMR: } n=22(51.1 \%) ; \\
\text { late AMR: } n=7(28.0 \%)\end{array}$ & $\begin{array}{l}\text { Decreased post-AMR } \\
\text { survival in patients with } \\
\text { late vs. early AMR: } 80 \\
\text { vs. } 93 \%, 1 \text { y; } 51 \text { vs. } \\
73 \%, 5 \text { y }(p<0.05)\end{array}$ & $\begin{array}{l}n=68(9.9 \%): 43 \text { early } \\
(<1 \text { y post- }-T x), 25 \text { late } \\
(>1 \text { y post }-T x)\end{array}$ & $\begin{array}{l}\text { No difference in } \\
\text { prevalence early } \\
\text { AMR vs. late } \\
\text { AMR }(p=0.51) \text {; } \\
\text { accelerated de } \\
\text { novo CAV in late } \\
\text { AMR + graft } \\
\text { dysfunction } \\
(50 \% \text { at } 1 \text { y, } \\
\text { HR }=5.42 \\
p=0.009)\end{array}$ & $\begin{array}{l}\text { Graft dysfunction increased in } \\
\text { late AMR group ( } 56.0 \text { vs. } 25.6 \% \text {, } \\
p=0.01 \text { ) }\end{array}$ \\
\hline
\end{tabular}

Ab, antibody; ACR, acute cellular rejection; AHG, anti-human globulin; AMR, antibody-mediated rejection; C19, complement component 19; C4d, complement component 4d; CAV, cardiac allograft vasculopathy; CDC, complementdependent cytotoxicity; CMR, cell-mediated rejection; XM, crossmatch; DSA, donor-specific HLA antibodies; dnDSA, de novo donor-specific HLA antibody; EMB, endomyocardial biopsies; GS, graft survival; HR, hazard ratio; IF, immunofluorescence; MFI, mean fluorescence intensity; MI, microcirculation inflammation; pAMR, pathologic AMR; post-TX, posttransplant; PRA, panel-reactive antibodies; pre-TX, pretransplant; SAB, Luminex single antigen bead assay; VLR, very late rejection; y, year(s); HLA, human leukocyte antigens. 
TABLE 2 | Cited publications from the last 6 years (2010-present) showing the impact of HLA antibody on heart transplantation in pediatric recipients.

\begin{tabular}{|c|c|c|c|c|c|c|c|}
\hline Reference & $\begin{array}{l}\text { Number of patients } \\
\text { (study period) }\end{array}$ & Method & DSA & GS & AMR & CAV & Comments \\
\hline $\begin{array}{l}\text { Rossano et al. } \\
\text { (31) }\end{array}$ & $\begin{array}{l}\text { 3,534 (October 1987- } \\
\text { May 2004, follow-up } \\
\text { through May 2008), } \\
\text { UNOS database }\end{array}$ & $\begin{array}{l}\text { CDC-PRAXXM most } \\
\text { commonly used }\end{array}$ & $\begin{array}{l}\text { PRA }>10 \%=387(11 \%) ; \\
9 \% \text { XM+ }\end{array}$ & $\begin{array}{l}\text { Median graft survival } \\
\text { PRA }>10 \%=7.1 y \\
\text { PRA } 1-10 \%=9.6 y \\
\text { PRA } 0 \%=9.8 y\end{array}$ & & & $\begin{array}{l}\text { Decreased long-term GS in } \\
\text { patients with PRA }>10 \%\end{array}$ \\
\hline Irving et al. (38) & $\begin{array}{l}\text { 59, mean post-Tx } \\
\text { follow-up } 5.1 \text { y (range } \\
0.7-18.5 \text { y) }\end{array}$ & Luminex screen/SAB & $\begin{array}{l}N=4(7 \%): 1 \text { transient } \\
\text { Class I, } 3 \text { persistent } \\
\text { Class II }\end{array}$ & $\begin{array}{l}\text { DSA+: } 1 / 4 \text { functioning, } \\
2 / 4 \text { retransplanted, and } \\
1 / 4 \text { died ( } 7 \text { y post-Tx) }\end{array}$ & $\begin{array}{l}\text { DSA+: } 2 / 4(50 \%) ; \\
\text { non-DSA+: } 1 / 15(7 \%) ; \\
\text { no Ab: } 5 / 40(13 \%)\end{array}$ & $\begin{array}{l}\text { DSA+: } 3 / 4 \text { (75\%); } \\
\text { non-DSA+: } 1 / 15 \\
\text { (7\%); no Ab: } 3 / 40 \\
(7.5 \%)\end{array}$ & $\begin{array}{l}\text { Severe cellular rejection ( } \geq 3 \mathrm{R}) \\
n=3(5.1 \%) \text {, all DSA- }\end{array}$ \\
\hline Chin et al. (42) & $\begin{array}{l}18 \text { (June 2007- } \\
\text { February 2009) }\end{array}$ & $\begin{array}{l}\text { CDC-XM, SAB, SAB-C1q, } \\
\text { Flow CXM }\end{array}$ & $\begin{array}{l}\text { SAB-IgG DSA: } \\
\text { Pre-Tx 61.1\%, } \\
\text { Post-Tx 55.5\%; } \\
\text { SAB-C19 DSA: Pre-Tx } \\
21.4 \% \text {, post-Tx } 35.7 \%\end{array}$ & 94\% (1 y), 82\% (2 y) & $\begin{array}{l}\text { Within 1st month: } \\
n=5 \text { (27.7\%), } \\
\text { all post-Tx SAB-C1q+ } \\
\text { DSA }\end{array}$ & & $\begin{array}{l}\text { SAB-C1q assay may better } \\
\text { predict early AMR }\end{array}$ \\
\hline Mahle et al. (32) & $\begin{array}{l}\text { 1,904 (January 1993- } \\
\text { December 2008) } \\
\text { Pediatric Heart } \\
\text { Transplant Study Group }\end{array}$ & $\begin{array}{l}\text { CDC-PRA most commonly } \\
\text { used }\end{array}$ & $\begin{array}{l}\text { PRA } \geq 10 \%=397 \\
(15.8 \%) \\
\text { PRA } \geq 50 \%= \\
189(7.6 \%)\end{array}$ & $\begin{array}{l}1 \text { y patient survival: } \\
\text { PRA } \geq 50 \%, 73 \text { vs. } \\
90 \% \text { for PRA }<10 \%\end{array}$ & & $\begin{array}{l}\text { No CAV association } \\
\text { with pre-Tx Ab }\end{array}$ & $\begin{array}{l}\text { No association of PRA with } \\
\text { time to } 1 \text { st rejection or CAV }\end{array}$ \\
\hline Ho et al. (16) & $\begin{array}{l}108 \text { (January 2000- } \\
\text { December 2009) }\end{array}$ & CDC-PRA, SAB & $\begin{array}{l}\text { PRA }>10 \% \\
\text { Class I }=9 \% \\
\text { Class } \|=14 \%\end{array}$ & $\begin{array}{l}87 \% \text { GS in CDC- vs. } \\
33 \% \text { CDC+ after } 7 y\end{array}$ & & & $\begin{array}{l}\text { Correlation between AMR and } \\
\text { presence of CDC- or SPA- } \\
\text { detected DSA }\end{array}$ \\
\hline Scott et al. (34) & 101 (2004-2008) & CDC-PRA, FLOW & & $\begin{array}{l}\text { PRA }>25 \% \text { decreased } \\
\text { GS vs. patients with } \\
\text { PRA }<25 \%\end{array}$ & $\begin{array}{l}n=12: 33 \% \text { with PRA } \\
>80 \% \text { vs. } 13 \% \text { with } \\
\text { PRA < } 80 \%\end{array}$ & & \\
\hline Peng et al. (44) & $\begin{array}{l}60 \text { (October 2005- } \\
\text { January 2011) }\end{array}$ & $\begin{array}{l}\text { FLOW-PRA, SAB, } 183 \\
\text { paired DSA and C4d }\end{array}$ & & & $6(3 / 6 \mathrm{XM}+)$ & & $\begin{array}{l}\text { Correlation between C4d+ in } \\
\text { EMB and DSA >6,000 MFI }\end{array}$ \\
\hline Daly et al. (58) & $\begin{array}{l}134 \text { (January 1998- } \\
\text { January 2011) }\end{array}$ & $\begin{array}{l}\text { CDC-AHG PRA, Luminex } \\
\text { SAB; XM+ patients } \\
\text { received preoperative } \\
\text { plasmapheresis + IVIG }\end{array}$ & $\begin{array}{l}12 \mathrm{XM}+(9 \%) \\
\mathrm{T}+/ \mathrm{B}+=8 \\
\mathrm{~T}-/ \mathrm{B}+=2 \\
\mathrm{~T}+/ \mathrm{B} \text { not tested }=2\end{array}$ & $\begin{array}{l}\text { No significant } \\
\text { difference in GS for } \\
\text { XM+ }(n=3,25 \%) \text { vs. } \\
X M-(n=12,10 \%)\end{array}$ & $\begin{array}{l}1 \mathrm{yr} \text { post-Tx: } \\
\mathrm{XM}+=6(50 \%) \\
\mathrm{XM}-=2(2 \%) \\
(p<0.001)\end{array}$ & & $\begin{array}{l}\text { Serious infection higher } \\
\text { in } X M+\text { vs. } X M-(50 \text { vs. } \\
16 \%, p=0.005) \text {; shorter } \\
\text { time to } 1 \text { st infection in } X M+ \\
(p=0.001)\end{array}$ \\
\hline $\begin{array}{l}\text { Asante-Korang } \\
\text { et al. (35) }\end{array}$ & $\begin{array}{l}70 \text { (January 2005- } \\
\text { July 2013) }\end{array}$ & $\begin{array}{l}\text { Luminex PRA, SAB, } \\
\text { Flow-XM; desensitization } \\
\text { performed in patients with } \\
\text { PRA }>10 \%\end{array}$ & PRA $>10 \%=14(20 \%)$ & $\begin{array}{l}\text { Overall patient survival: } \\
92.9 \% \text { in sensitized } \\
\text { group vs. } 80.4 \% \text { in } \\
\text { non-sensitized }\end{array}$ & $\begin{array}{l}\text { Freedom from AMR } \\
\text { or rejection grade } \\
\geq 2 R / 3 A: 71.4 \% \text { in } \\
\text { sensitized vs. } 64 \% \text { in } \\
\text { non-sensitized }\end{array}$ & $\begin{array}{l}\text { Freedom from } \\
\text { CAV: } 93 \% \text { for } \\
\text { sensitized vs. } 91 \% \text { in } \\
\text { non-sensitized }\end{array}$ & $\begin{array}{l}\text { 12/14 high PRA patients had } \\
\text { reduced Ab levels following } \\
\text { desensitization; no significant } \\
\text { differences in outcomes } \\
\text { between desensitized patients } \\
\text { and those with no Ab }\end{array}$ \\
\hline
\end{tabular}




\section{TABLE 2 | Continued}

\begin{tabular}{|c|c|c|c|c|c|c|c|}
\hline Reference & $\begin{array}{l}\text { Number of patients } \\
\text { (study period) }\end{array}$ & Method & DSA & GS & AMR & CAV & Comments \\
\hline Chen et al. (36) & $\begin{array}{l}25 \text { (January 2008- } \\
\text { June 2010) }\end{array}$ & PRA and SAB, 195 samples & $12 / 25 \mathrm{dnDSA}$ & $\begin{array}{l}\text { No impact short-term } \\
\text { survival }\end{array}$ & & & Majority of dnDSA within $1 \mathrm{y}$ \\
\hline Irving et al. (47) & 108 (1996-2009) & SAB, 691 samples & $\begin{array}{l}43 \text { DSA ( } 58 \% \text { persistent) } \\
\text { Class I }=30 \% \\
\text { Class } \|=47 \% \\
\text { Class I }+\|=23 \%\end{array}$ & $\begin{array}{l}\text { 9/14 with graft loss had } \\
\text { persistent DSA }\end{array}$ & & $\begin{array}{l}\text { 9/10 with CAV } \\
\text { DSA+; 6/9 DSA } \\
\text { persistent }\end{array}$ & $\begin{array}{l}\text { Persistent DSA associated } \\
\text { with poor outcome and CAV }\end{array}$ \\
\hline $\begin{array}{l}\text { Godown } \\
\text { et al. (39) }\end{array}$ & $\begin{array}{l}121 \text { (1987-2014), } \\
\text { mean follow-up } 4.1 \text { y }\end{array}$ & $\begin{array}{l}\text { Flow, Luminex, } \\
\text { all were XM- }\end{array}$ & $\begin{array}{l}\text { dnDSA: } 40(33 \%) \\
\text { Class I = } 24 \% \\
\text { Class } I=50 \% \\
\text { Class I + II = 26\% }\end{array}$ & & & & $\begin{array}{l}\text { Multiple factors influence DSA } \\
\text { development; DSA seen more } \\
\text { frequently in patients with prior } \\
\text { sensitizing events }\end{array}$ \\
\hline Ware et al. (43) & $\begin{array}{l}66 \text { (January 2009- } \\
\text { September 2013) }\end{array}$ & $\mathrm{SAB}$ & $27 \mathrm{DSA}+(4 \mathrm{XM}+)$ & No impact & $\begin{array}{l}\text { DSA level associated } \\
\text { with pAMR2, } 3\end{array}$ & No impact & $\begin{array}{l}\text { Negative predictive value of } \\
\text { DSA testing for absence of } \\
\text { pAMR }\end{array}$ \\
\hline Tran et al. (37) & $\begin{array}{l}105 \text { (January 2002- } \\
\text { December 2012, } \\
\text { follow-up 0.13-10.8 y) }\end{array}$ & $\begin{array}{l}\text { SAB ( } 5 \text { times first year } \\
\text { and yearly after) }\end{array}$ & $\begin{array}{l}45(43 \%) \text { DSA } \\
\text { Class I = } 20 \% \\
\text { Class } I=62.2 \% \\
\text { Class I }+\|=17.8 \%\end{array}$ & $\begin{array}{l}5 \text { y GS } 72.4 \% \text { DSA- } \\
\text { vs. } 21 \% \text { DSA+ }\end{array}$ & & $\begin{array}{l}\text { CAV 36\% DSA+ vs. } \\
13 \% \text { DSA- }\end{array}$ & $\begin{array}{l}\text { DSA+ had } 2.5 \text { times more } \\
\text { rejection events per year } \\
\text { compared to DSA- }\end{array}$ \\
\hline $\begin{array}{l}\text { Thrush } \\
\text { et al. (40) }\end{array}$ & $\begin{array}{l}\text { 1,596 (January 2010- } \\
\text { December 2014), } \\
\text { Pediatric Heart } \\
\text { Transplant Study } \\
\text { database }\end{array}$ & Unknown & & $\begin{array}{l}33 \text { deaths (16\%) } \\
\text { post-AMR } \\
\text { development; } \\
\text { short-term patient/ } \\
\text { GS lower for patients } \\
\text { with treated AMR } \\
(p=0.004, p=0.001 \text {, } \\
\text { respectively); patient } \\
\text { survival post-AMR } \\
\text { diagnosis: } 88 \% 1 \mathrm{y}, \\
77 \% 3 \mathrm{y}\end{array}$ & $\begin{array}{l}179(11 \%), \text { freedom } \\
\text { from AMR: } 88 \% 1 \text { y, } \\
82 \% 3 y\end{array}$ & & $\begin{array}{l}\text { AMR often concurrent with } \\
\text { ACR }\end{array}$ \\
\hline
\end{tabular}

Ab, antibody; ACR, acute cellular rejection; AHG, anti-human globulin; AMR, antibody-mediated rejection; C1q, complement component 19; C4d, complement component 4d; cAMR, clinical AMR; CAV, cardiac allograft vasculopathy: CDC, complement-dependent cytotoxicity; XM, crossmatch; DSA, donor-specific HLA antibodies; dnDSA, de novo donor-specific HLA antibody; EMB, endomyocardial biopsies; GS, graft survival; HR, hazard ratio; IF, immunofluorescence; pAMR, pathologic AMR; post-Tx, posttransplant; PRA, panel-reactive antibodies; pre-Tx, pretransplant; SAB, Luminex single antigen bead assay; SPA, solid phase assays; $y$, year(s); MFI, mean fluorescence intensity; HLA, human leukocyte antigens. 
complement-fixing reactivity $(7,20)$ and has been used to determine unacceptable HLA antigens for sensitized candidates (5). DSA determination by SPA and elevated peak panel-reactive antibodies (PRA) were independent predictors of pAMR in an adult cohort of heart transplant recipients (21). In this study focusing on pretransplant samples, increasing numbers of DSAs and the mean cumulative MFI of DSAs were associated with risk of AMR, and the subset of C1q-reactive DSAs were less informative (21). Pathologic classification of AMR in 37 EMB correlated with circulating DSA and endothelial activation (22). The proportion of DSA+ EMB varied according to pAMR grade, and pAMR2 was associated with $100 \%$ DSA positivity (22). The clinical significance of DSA level as depicted only by MFI of neat serum is still controversial, and currently multiple approaches are proposed to capture the DSA level and function, including serum titration and complement-binding assays. A better concordance was observed between C1q+DSA and C4d immunofluorescence (IF)+ staining in EMB as compared with total IgG DSAs and C4d IF+ in EMB among 44 recipients (40 vs. $24 \%, p=0.02)(23)$. A majority ( $82 \%)$ of patients with graft dysfunction had circulating C1q+ DSAs (23). However, not all patients with circulating $\mathrm{C} 1 \mathrm{q}+\mathrm{DSA}$ had $\mathrm{C} 4 \mathrm{~d} \mathrm{IF}+$ staining on $\mathrm{EMB}$, suggesting that the presence of $\mathrm{C} 1 \mathrm{q}+\mathrm{DSA}$ may precede the development of pAMR or be due to the low sensitivity of C4d IF staining (23).

Prognosis after late AMR (defined as AMR >1 year posttransplant) was poor in 20 recipients despite aggressive treatment with immunosuppression, and fulminant CAV was a common condition (24). DSA was present in all tested patients $(n=19)$ with a median cumulative MFI at diagnosis $>10,000$; most of the patients had de novo DSA (24). Antibody-mediated injury and immune-mediated coronary arteriosclerosis were the causes of late graft failure in a recent study of 40 explanted heart allografts (25). AMR was observed in 47.5\% failing heart allografts, including $40 \%$ of patients in whom unrecognized previous episodes of subclinical AMR occurred years before allograft loss. Among the 19 patients with AMR, 15 were tested for DSA, and 93\% had circulating DSA at the time of allograft failure. The immunodominant DSA was Class II in 11/14 DSA+, and the median DSA MFI was 5,000 (25). In contrast, only $37 \%$ of patients without AMR features at the time of allograft failure had circulating DSA as compared to the AMR group $(p<0.001)$, and the median DSA MFI was 1,250 (25). In a retrospective cohort study spanning over 10 years, the timing of AMR (early vs. late) was associated with GS and CAV (26). Patients were tested at the time of biopsy for circulating DSA either by CDC (before 2010) or SAB (post 2010). Graft dysfunction was less frequent in early AMR, while late AMR with graft dysfunction showed rapid development of de novo CAV despite aggressive treatment and also increased risk of death (26).

\section{Cardiac Vasculopathy}

Cardiac allograft vasculopathy continues to remain a limiting factor in long-term survival of heart transplant recipients, and there is increasing evidence of the negative impact of circulating DSA on the development and severity of CAV. Patients with DSA had significantly higher rates and a shorter mean time to
CAV and increased severity of CAV as compared to patients without DSA $(27,28)$. Patients with very late rejection and circulating DSA with evidence of intravascular macrophages had an increased risk of severe CAV as compared to patients without DSA (29).

\section{PEDIATRIC HT}

\section{Graft Survival}

Allosensitization and GS in pediatric HT recipients have been evaluated in large single- (30) and multicenter datasets $(31,32)$ (Table 2). Pediatric patients with PRA $>10 \%$ had earlier-onset graft vasculopathy (30) and worse graft and patient survival than did patients with PRA $<10 \%(31,32)$. Elevated PRA was an independent risk factor for worse long-term GS (31). Furthermore, significant allosensitization (PRA >50\%) at listing was associated with a more than twofold increased risk of death within the first transplant year (32). These large patient cohorts that were transplanted over a period of 18 years may have underestimated the rate of allosensitization because the methodology for PRA screening evolved from a less sensitive cell-based method to the more sensitive SPA. In addition, the SPA may have also increased the need for prospective XM due to an increased use of VXM (33).

In a more recent study patients with PRA $>25 \%$ had significantly $(p=0.004)$ decreased survival compared to those with PRA $<25 \%$ (34). In contrast, the outcome of allosensitized pediatric patients with PRA $>10 \%$ who were desensitized was not different than non-sensitized recipients (35).

Assessments of GS in the presence of DSA show somewhat mixed findings, perhaps related to the duration of follow-up. Although short-term GS was not impacted by the presence of DSA in one pediatric study (36), the 5-year survival was significantly better in patients without DSA in another pediatric cohort (72 vs. 21\%) (37). While uncommon, the presence of de novo DSA posttransplantation, especially toward Class II HLA, was associated with increased graft loss (38).

Multiple factors appear to play a role in development of de novo DSA in pediatric HT including prior sensitizing events, older age, African-American race, and donor death from gunshot wound (39). Knowledge of risk factors for the development of de novo DSA in pediatric recipients is likely to be important to guide the frequency of monitoring for HLA antibodies (39).

\section{Antibody-Mediated Rejection}

Current understanding of AMR after HT is largely derived from adult studies. Using the Pediatric Heart Transplant Study database, the reported incidence of AMR was 11\% (among 1,596 recipients), and patient and GS were lower for those with AMR (40). Risk factors associated with AMR included PRA $>10 \%$ at $\mathrm{HT}$, a positive CDC XM, and congenital heart disease, suggesting allosensitization related to the use of homografts (40).

The proportion of AMR-free patients was much higher among patients with only solid phase-detected DSA vs. those with CDCdetected DSA (41). Similarly, using the C1q assay, which detects only complement-fixing antibodies, the presence of $\mathrm{Clq}$ fixing DSA prior to or early after HT had a positive predictive value of 
$100 \%$, while absence of C1q fixing DSA had a negative predictive value of $100 \%$ for AMR (42). In another analysis, the presence of circulating DSA had $93 \%$ sensitivity, $62 \%$ specificity, $24 \%$ positive predictive value, and $99 \%$ negative predictive value for biopsy diagnosis of AMR in pediatric recipients (43). In addition, higher levels of circulating DSA measured by MFI correlated with pAMR severity (43). The authors proposed that DSA monitoring provides a non-invasive tool to tailor the frequency of biopsy surveillance (43). Others have used an institution-specific MFI threshold value for DSA of $>6,000$ that strongly correlated with C4d deposition on EMB with high negative predictive value (97\%) and specificity (95\%) (44). Both studies emphasized the advantage of following DSA in asymptomatic pediatric patients, given the value of early detection of $\operatorname{AMR}(43,44)$.

Similar to findings for renal transplantation (45), sensitized recipients with persistent posttransplant DSA with complementfixing ability appear to be at high risk for $\operatorname{AMR}(20,42,46)$.

\section{Cardiac Vasculopathy}

Overall, DSA+ patients (preformed or de novo) had significantly higher rates of CAV compared with DSA- patients. By 5 years, the rate of CAV-free survival was $25 \%$ for DSA- vs. $0 \%$ for DSA+ (37). Persistent DSA was associated with poor outcome and development of CAV (47).

\section{TREATMENT}

Desensitization is aimed to increase the donor pool by either reducing or eliminating HLA allosensitization or by facilitating transplant by reducing the DSA burden. Desensitization treatment targets critical components of the humoral response to either achieve a negative crossmatch pretransplant or to reduce the impact of DSA in positive crossmatch transplants. At low titer, antibody reduction can be achieved with plasma exchange and IVIG. The use of B cell suppression agents (rituximab), plasma cell depletion agents (bortezomib), or inhibitors of complement activation (eculizumab) is usually limited to highly sensitized patients. The current literature in adults is not abundant, mostly observational, with small cohorts, short follow-up, and with inconsistent treatment methodologies (48-53). In 21 highly sensitized patients, the use of plasmapheresis (PP), IVIG, rituximab, and cyclophosphamide resulted in comparable long-term survival when compared to the low sensitized and unsensitized cohorts (53). A recent experience with bortezomib and PP showed that about $50 \%$ of the patients had a calculated PRA reduction and were transplanted with a negative crossmatch $(48,52)$. One year follow-up showed $100 \%$ survival and $74 \%$ freedom from rejection $(48,52)$. In a smaller cohort of patients transplanted across a positive crossmatch and treated with eculizumab and ATG, 1-year survival was $89 \%$, and freedom from rejection was $75 \%$ (52). In patients treated for AMR, Class I HLA antibodies demonstrated a statistically significant response to bortezomib, whereas Class II responded poorly (51).

In pediatric $\mathrm{HT}$, requiring a negative prospective crossmatch increases the waiting time and more importantly the waitlist mortality (10). Allosensitization is most significant among children with certain forms of congenital heart disease due to the use of homograft during prior surgeries. Also, blood transfusions and VAD use are common causes of allosensitization. Current literature for pediatric heart transplant desensitization is even more limited than in the adult cohort (54-58). Desensitization was carried out successfully with bortezomib and PP in a pediatric setting (54). Furthermore, in a single-center retrospective study in a large cohort of patients, all sensitized patients received PP or plasma exchange preoperatively. If the cytotoxic XM was positive, $\mathrm{PP}$ was continued. Patients with negative $\mathrm{XM}$ did not receive additional PP and IVIG posttransplant (58). Hemodynamically significant AMR occurred in 50\% of patients transplanted across a positive XM vs. $2 \%$ of the XM-negative cohort (58). Additionally, incidence of serious infection was higher in patients transplanted across a positive crossmatch (58). Antibody depletion therapies were also used in management of AMR in pediatric patients. Decreased DSA MFI in 21 patients treated with PP correlated with good clinical outcome (55). In another small study, addition of bortezomib to PP and rituximab treatment resulted in a rapid decline in DSA and reversal of AMR without significant side effects (56).

\section{SUMMARY}

The negative impact of DSA on patient and allograft survival is evident in adult and pediatric HT recipients. Allosensitization depicted by PRA $>10 \%$ using cell based (prior era) or SPA (current era) is associated with poor outcome in both cohorts. Furthermore, similar risk factors were identified in adults and pediatric recipients for the development of posttransplant DSA including sensitizing events pretransplant, ECMO, need for mechanical support, non-compliance, and African-American race. In adults, but not in pediatrics, female gender (prior pregnancies) was also associated with a higher risk for development of de novo DSA. In children, exposure to homografts as part of surgical repair for some forms of congenital heart disease increase their risk for allosensitization and AMR.

Many questions remain regarding the most appropriate frequency of assessment of pre- and posttransplant DSA as well as the phenotype of DSA memory vs. true de novo antibody using large multicenter adult and pediatric cohorts and state-of-theart methodologies for DSA detection and characterization. The observation that early vs. late AMR in HT may have different prognosis and responses to treatment emphasizes the need to assess the risk of sensitization pretransplantation and to follow by routine monitoring of DSA posttransplant.

The ongoing multicenter clinical collaborative studies supported by National Institute of Health in adult and pediatric HT will hopefully provide answers to many remaining questions regarding the impact of preformed and de novo DSA on clinical outcomes and the efficacy of various modalities for desensitization and treatment of AMR.

\section{AUTHOR CONTRIBUTIONS}

This is an invited review on impact of HLA donor-specific antibody in cardiac allograft outcome. All authors contributed equally to literature review, summary, and manuscript preparation. 


\section{REFERENCES}

1. Tait BD, Süsal C, Gebel HM, Nickerson PW, Zachary AA, Claas FHJ, et al. Consensus guidelines on the testing and clinical management issues associated with HLA and non-HLA antibodies in transplantation. Transplantation (2013) 95(1):19-47. doi:10.1097/TP.0b013e31827a19cc

2. Campbell P. Clinical relevance of human leukocyte antigen antibodies in liver, heart, lung and intestine transplantation. Curr Opin Organ Transplant (2013) 18(4):463-9. doi:10.1097/MOT.0b013e3283636c71

3. Picascia A, Grimaldi V, Casamassimi A, De Pascale MR, Schiano C, Napoli C. Human leukocyte antigens and alloimmunization in heart transplantation: an open debate. J Cardiovasc Transl Res (2014) 7(7):664-75. doi:10.1007/ s12265-014-9587-z

4. Eckels DD, Stehlik J, Kfoury AG. The detection and role of circulating antibodies in rejection. Curr Opin Organ Transplant (2013) 18(5):589-94. doi:10.1097/MOT.0b013e328364fe3d

5. Reinsmoen NL, Patel J, Mirocha J, Lai CH, Naim M, Ong G, et al. Optimizing transplantation of sensitized heart candidates using 4 antibody detection assays to prioritize the assignment of unacceptable antigens. J Heart Lung Transplant (2016) 35(2):165-72. doi:10.1016/j.healun.2015.10.013

6. Castleberry C, Ryan TD, Chin C. Transplantation in the highly sensitized pediatric patient. Circulation (2014) 129(22):2313-9. doi:10.1161/ CIRCULATIONAHA.113.001378

7. Tambur AR, Herrera ND, Haarberg KM, Cusick MF, Gordon RA, Leventhal JR, et al. Assessing antibody strength: comparison of MFI, C1q, and Titer Information. Am J Transplant (2015) 15(9):2421-30. doi:10.1111/ ajt.13295

8. Tambur AR, Glotz D, Herrera ND, Chatroop EN, Roitberg T, Friedewald JJ, et al. Can solid phase assays be better utilized to measure efficacy of antibody removal therapies? Hum Immunol (2016) 77(8):624-30. doi:10.1016/j. humimm.2016.05.025

9. Otten HG, Verhaar MC, Borst HP, van Eck M, van Ginkel WG, Hené RJ, et al. The significance of pretransplant donor-specific antibodies reactive with intact or denatured human leucocyte antigen in kidney transplantation. Clin Exp Immunol (2013) 173(3):536-43. doi:10.1111/cei.12127

10. Feingold B, Park SY, Comer DM, Moore CG, Webber SA, Bryce CL. Outcomes after listing with a requirement for a prospective crossmatch in pediatric heart transplantation. J Heart Lung Transplant (2013) 32(1):56-62. doi:10.1016/j. healun.2012.09.023

11. Potena L, Bontadini A, Iannelli S, Fruet F, Leone O, Barberini F, et al. Occurrence of fatal and nonfatal adverse outcomes after heart transplantation in patients with pretransplant noncytotoxic HLA antibodies. J Transplant (2013) 2013:519680. doi:10.1155/2013/519680

12. O'Connor MJ, Keeshan BC, Lin KY, Monos D, Lind C, Paridon SM, et al. Changes in the methodology of pre-heart transplant human leukocyte antibody assessment: an analysis of the United Network for Organ Sharing database. Clin Transplant (2015) 29(9):842-50. doi:10.1111/ctr.12590

13. Raess M, Fröhlich G, Roos M, Rüsi B, Wilhelm MJ, Noll G, et al. Donorspecific anti-HLA antibodies detected by Luminex: predictive for short-term but not long-term survival after heart transplantation. Transpl Int (2013) 26(11):1097-107. doi:10.1111/tri.12170

14. Smith JD, Banner NR, Hamour IM, Ozawa M, Goh A, Robinson D, et al. De novo donor HLA-specific antibodies after heart transplantation are an independent predictor of poor patient survival. Am J Transplant (2011) 11(2):312-9. doi:10.1111/j.1600-6143.2010.03383.x

15. Hodges AM, Lyster H, McDermott A, Rice AJ, Smith JD, Rose ML, et al. Late antibody-mediated rejection after heart transplantation following the development of de novo donor-specific human leukocyte antigen antibody. Transplantation (2012) 93(6):650-6. doi:10.1097/TP.0b013e318244f7b8

16. Ho EK, Vlad G, Vasilescu ER, de la Torre L, Colovai AI, Burke E, et al. Pre- and posttransplantation allosensitization in heart allograft recipients: major impact of de novo alloantibody production on allograft survival. Hum Immunol (2011) 72(1):5-10. doi:10.1016/j.humimm.2010.10.013

17. Kobashigawa J, Crespo-Leiro MG, Ensminger SM, Reichenspurner H, Angelini A, Berry G, et al. Report from a consensus conference on antibodymediated rejection in heart transplantation. J Heart Lung Transplant (2011) 30(3):252-69. doi:10.1016/j.healun.2010.11.003

18. Kittleson MM, Kobashigawa JA. Antibody-mediated rejection. Curr Opin Organ Transplant (2012) 17(5):551-7. doi:10.1097/MOT.0b013e3283577fef
19. Gandhi MJ, DeGoey SR, Bundy K, Kremers WK, Knauer R, Pereira N, et al. Effect of pretransplant human leukocyte antigen antibodies detected by solid-phase assay on heart transplant outcomes. Transplant Proc (2011) 43(10):3840-6. doi:10.1016/j.transproceed.2011.08.077

20. Zeevi A, Lunz J, Feingold B, Shullo M, Bermudez C, Teuteberg J, et al. Persistent strong anti-HLA antibody at high titer is complement binding and associated with increased risk of antibody-mediated rejection in heart transplant recipients. J Heart Lung Transplant (2013) 32(1):98-105. doi:10.1016/j. healun.2012.09.021

21. Svobodova E, Gazdic T, Kubanek M, Vymetalova J, Voska L, Kment M, et al. Novel insights into pretransplant allosensitization in heart transplant recipients in the contemporary era of immunosuppression and rejection surveillance. Transpl Int (2016) 29(1):63-72. doi:10.1111/tri.12684

22. Tible M, Loupy A, Vernerey D, Suberbielle C, Beuscart T, Cazes A, et al. Pathologic classification of antibody-mediated rejection correlates with donor-specific antibodies and endothelial cell activation. J Heart Lung Transplant (2013) 32(8):769-76. doi:10.1016/j.healun.2013.05.012

23. Frank R, Lal P, Kearns J, Molina MR, Wald JW, Goldberg LR, et al. Correlation of circulating complement-fixing donor-specific antibodies identified by the $\mathrm{C} 1 \mathrm{q}$ assay and presence of $\mathrm{C} 4 \mathrm{~d}$ in endomyocardial biopsy specimens. Am J Clin Pathol (2016) 145(1):62-8. doi:10.1093/ajcp/aqv016

24. Coutance G, Ouldamar S, Rouvier P, Saheb S, Suberbielle C, Bréchot N, et al. Late antibody-mediated rejection after heart transplantation: mortality, graft function, and fulminant cardiac allograft vasculopathy. J Heart Lung Transplant (2015) 34(8):1050-7. doi:10.1016/j.healun.2015.03.002

25. Loupy A, Toquet C, Rouvier P, Beuscart T, Bories MC, Varnous S, et al. Late failing heart allografts: pathology of cardiac allograft vasculopathy and association with antibody-mediated rejection. Am J Transplant (2016) 16(1):111-20. doi:10.1111/ajt.13529

26. Clerkin KJ, Restaino SW, Zorn E, Vasilescu ER, Marboe CC, Mancini DM. The effect of timing and graft dysfunction on survival and cardiac allograft vasculopathy in antibody-mediated rejection. J Heart Lung Transplant (2016) 35(9):1059-66. doi:10.1016/j.healun.2016.04.007

27. Topilsky Y, Gandhi MJ, Hasin T, Voit LL, Raichlin E, Boilson BA, et al. Donor-specific antibodies to class II antigens are associated with accelerated cardiac allograft vasculopathy: a three-dimensional volumetric intravascular ultrasound study. Transplantation (2013) 95(2):389-96. doi:10.1097/ TP.0b013e318273878c

28. Frank R, Molina MR, Goldberg LR, Wald JW, Kamoun M, Lal P. Circulating donor-specific anti-human leukocyte antigen antibodies and complement C4d deposition are associated with the development of cardiac allograft vasculopathy. Am JClin Pathol (2014) 142(6):809-15. doi:10.1309/ AJCPTLBEU5BQ8SHN

29. Loupy A, Cazes A, Guillemain R, Amrein C, Hedjoudje A, Tible M, et al. Very late heart transplant rejection is associated with microvascular injury, complement deposition and progression to cardiac allograft vasculopathy. Am J Transplant (2011) 11(7):1478-87. doi:10.1111/j.1600-6143.2011.03563.x

30. Feingold B, Bowman P, Zeevi A, Girnita AL, Quivers ES, Miller SA, et al. Survival in allosensitized children after listing for cardiac transplantation. J Heart Lung Transplant (2007) 26(6):565-71. doi:10.1016/j.healun.2007.03.015

31. Rossano JW, Morales DLS, Zafar F, Denfield SW, Kim JJ, Jefferies JL, et al. Impact of antibodies against human leukocyte antigens on long-term outcome in pediatric heart transplant patients: an analysis of the United Network for Organ Sharing database. J Thorac Cardiovasc Surg (2010) 140(3):694-9, 699. e1-2. doi:10.1016/j.jtcvs.2010.04.009

32. Mahle WT, Tresler MA, Edens RE, Rusconi P, George JF, Naftel DC, et al. Allosensitization and outcomes in pediatric heart transplantation. J Heart Lung Transplant (2011) 30(11):1221-7. doi:10.1016/j.healun.2011. 06.005

33. Feingold B, Park SY, Comer DM, Bryce CL, Webber SA. Listing requirements for a prospective crossmatch in pediatric heart transplantation: analysis of Organ Procurement and Transplantation Network data from 1996 to 2009. J Heart Lung Transplant (2012) 31(10):1143-4. doi:10.1016/j. healun.2012.08.006

34. Scott V, Williams RJ, Levi DS. Outcomes of cardiac transplantation in highly sensitized pediatric patients. Pediatr Cardiol (2011) 32(5):615-20. doi:10.1007/ s00246-011-9928-5

35. Asante-Korang A, Amankwah EK, Lopez-Cepero M, Ringewald J, Carapellucci J, Krasnopero D, et al. Outcomes in highly sensitized pediatric 
heart transplant patients using current management strategies. J Heart Lung Transplant (2015) 34(2):175-81. doi:10.1016/j.healun.2014.09.027

36. Chen CK, Manlhiot C, Conway J, Allain-Rooney T, McCrindle BW, Tinckam $\mathrm{K}$, et al. Development and impact of de novo anti-HLA antibodies in pediatric heart transplant recipients. Am J Transplant (2015) 15(8):2215-22. doi:10.1111/ajt.13259

37. Tran A, Fixler D, Huang R, Meza T, Lacelle C, Das BB. Donor-specific HLA alloantibodies: impact on cardiac allograft vasculopathy, rejection, and survival after pediatric heart transplantation. J Heart Lung Transplant (2016) 35(1):87-91. doi:10.1016/j.healun.2015.08.008

38. Irving C, Carter V, Parry G, Hasan A, Kirk R. Donor-specific HLA antibodies in paediatric cardiac transplant recipients are associated with poor graft survival. Pediatr Transplant (2011) 15(2):193-7. doi:10.1111/j.1399-3046. 2010.01446.x

39. Godown J, Slaughter JC, Fossey SC, McKane M, Dodd DA. Risk factors for the development of donor-specific antibodies after pediatric heart transplantation. Pediatr Transplant (2015) 19(8):906-10. doi:10.1111/ petr.12606

40. Thrush PT, Pahl E, Naftel DC, Pruitt E, Everitt MD, Missler H, et al. A multi-institutional evaluation of antibody-mediated rejection utilizing the Pediatric Heart Transplant Study database: incidence, therapies and outcomes. J Heart Lung Transplant (2016) 35(12):1497-504. doi:10.1016/ j.healun.2016.06.014

41. Ho EK, Vlad G, Colovai AI, Vasilescu ER, Schwartz J, Sondermeijer H, et al. Alloantibodies in heart transplantation. Hum Immunol (2009) 70(10):825-9. doi:10.1016/j.humimm.2009.06.015

42. Chin C, Chen G, Sequeria F, Berry G, Siehr S, Bernstein D, et al. Clinical usefulness of a novel C1q assay to detect immunoglobulin G antibodies capable of fixing complement in sensitized pediatric heart transplant patients. J Heart Lung Transplant (2011) 30(2):158-63. doi:10.1016/j.healun.2010. 08.020

43. Ware AL, Malmberg E, Delgado JC, Hammond ME, Miller DV, Stehlik J, et al. The use of circulating donor specific antibody to predict biopsy diagnosis of antibody-mediated rejection and to provide prognostic value after heart transplantation in children. J Heart Lung Transplant (2016) 35(2):179-85. doi:10.1016/j.healun.2015.10.006

44. Peng DM, Law YM, Kemna MS, Warner P, Nelson K, Boucek RJ. Donorspecific antibodies: can they predict C4d deposition in pediatric heart recipients? Pediatr Transplant (2013) 17(5):429-35. doi:10.1111/petr.12075

45. Loupy A, Lefaucheur C, Vernerey D, Prugger C, Duong van Huyen JP, Mooney $\mathrm{N}$, et al. Complement-binding anti-HLA antibodies and kidney-allograft survival. N Engl J Med (2013) 369(13):1215-26. doi:10.1056/NEJMoa1302506

46. Chin C. Cardiac antibody-mediated rejection. Pediatr Transplant (2012) 16(5):404-12. doi:10.1111/j.1399-3046.2012.01690.x

47. Irving CA, Carter V, Gennery AR, Parry G, Griselli M, Hasan A, et al. Effect of persistent versus transient donor-specific HLA antibodies on graft outcomes in pediatric cardiac transplantation. J Heart Lung Transplant (2015) 34(10):1310-7. doi:10.1016/j.healun.2015.05.001

48. Patel J, Everly M, Chang D, Kittleson M, Reed E, Kobashigawa J. Reduction of alloantibodies via proteasome inhibition in cardiac transplantation.
J Heart Lung Transplant (2011) 30(12):1320-6. doi:10.1016/j.healun.2011. 08.009

49. Cole RM, Kobashigawa JA. Desensitization strategies pre- and post-cardiac transplantation. Curr Treat Options Cardiovasc Med (2016) 18(2):8. doi:10.1007/s11936-015-0431-9

50. Nair N, Ball T, Uber PA, Mehra MR. Current and future challenges in therapy for antibody-mediated rejection. J Heart Lung Transplant (2011) 30(6):612-7. doi:10.1016/j.healun.2011.02.002

51. Khuu T, Cadeiras M, Wisniewski N, Reed EF, Deng MC. Reduced HLA Class II antibody response to proteasome inhibition in heart transplantation. J Heart Lung Transplant (2015) 34(6):863-5. doi:10.1016/j.healun.2015.01.982

52. Chih S, Patel J.Desensitization strategies in adult heart transplantation-Will persistence pay off? J Heart Lung Transplant (2016) 35(8):962-72. doi:10.1016/j.healun.2016.03.021

53. Kobashigawa JA, Patel JK, Kittleson MM, Kawano MA, Kiyosaki KK, Davis SN, et al. The long-term outcome of treated sensitized patients who undergo heart transplantation. Clin Transplant (2011) 25(1):E61-7. doi:10.1111/j.1399-0012.2010.01334.x

54. May LJ, Yeh J, Maeda K, Tyan DB, Chen S, Kaufman BD, et al. HLA desensitization with bortezomib in a highly sensitized pediatric patient. Pediatr Transplant (2014) 18(8):E280-2. doi:10.1111/petr.12347

55. Jackups R Jr, Canter C, Sweet SC, Mohanakumar T, Morris GP. Measurement of donor-specific HLA antibodies following plasma exchange therapy predicts clinical outcome in pediatric heart and lung transplant recipients with antibody-mediated rejection. J Clin Apher (2013) 28(4):301-8. doi:10.1002/ jca. 21270

56. Morrow WR, Frazier EA, Mahle WT, Harville TO, Pye SE, Knecht KR, et al. Rapid reduction in donor-specific anti-human leukocyte antigen antibodies and reversal of antibody-mediated rejection with bortezomib in pediatric heart transplant patients. Transplantation (2012) 93(3):319-24. doi:10.1097/ TP.0b013e31823f7eea

57. Gambetta K, Tambur A, Pahl E. Immune monitoring of pediatric heart transplant recipients through serial donor specific antibody testing - an initial experience and review of the literature. Prog Pediatr Cardiol (2011) 32(1):43-9. doi:10.1016/j.ppedcard.2011.06.009

58. Daly KP, Chandler SF, Almond CS, Singh TP, Mah H, Milford E, et al. Antibody depletion for the treatment of crossmatch-positive pediatric heart transplant recipients. Pediatr Transplant (2013) 17(7):661-9. doi:10.1111/petr.12131

Conflict of Interest Statement: The authors declare that the research was conducted in the absence of any commercial or financial relationships that could be construed as a potential conflict of interest.

Copyright (๑ 2017 Mangiola, Marrari, Feingold and Zeevi. This is an open-access article distributed under the terms of the Creative Commons Attribution License (CC BY). The use, distribution or reproduction in other forums is permitted, provided the original author(s) or licensor are credited and that the original publication in this journal is cited, in accordance with accepted academic practice. No use, distribution or reproduction is permitted which does not comply with these terms. 